\title{
Intelligent control for modeling of real-time reservoir operation, part II: artificial neural network with operating rule curves
}

\author{
Ya-Ting Chang, Li-Chiu Chang and Fi-John Chang* \\ Department of Bioenvironmental Systems Engineering, National Taiwan University, Taipei, Taiwan
}

\begin{abstract}
:
To bridge the gap between academic research and actual operation, we propose an intelligent control system for reservoir operation. The methodology includes two major processes, the knowledge acquired and implemented, and the inference system. In this study, a genetic algorithm (GA) and a fuzzy rule base (FRB) are used to extract knowledge based on the historical inflow data with a design objective function and on the operating rule curves respectively. The adaptive network-based fuzzy inference system (ANFIS) is then used to implement the knowledge, to create the fuzzy inference system, and then to estimate the optimal reservoir operation. To investigate its applicability and practicability, the Shihmen reservoir, Taiwan, is used as a case study. For the purpose of comparison, a simulation of the currently used M-5 operating rule curve is also performed. The results demonstrate that (1) the GA is an efficient way to search the optimal input-output patterns, (2) the FRB can extract the knowledge from the operating rule curves, and (3) the ANFIS models built on different types of knowledge can produce much better performance than the traditional M-5 curves in real-time reservoir operation. Moreover, we show that the model can be more intelligent for reservoir operation if more information (or knowledge) is involved. Copyright (c) 2004 John Wiley \& Sons, Ltd.
\end{abstract}

KEY WORDS genetic algorithm; artificial neural network; fuzzy rule base; adaptive network-based fuzzy inference system; reservoir operation

\section{INTRODUCTION}

The main purpose of this paper is to enhance our previous work (Chang and Chang, 2001) through combining fuzzy operating rule curves into our original intelligent control system so that it will involve more knowledge, and to investigate its applicability and practicability for real-time operation of the Shihmen reservoir, Taiwan.

Real-time reservoir operation can be very complex because of the inherent variability of natural streamflow and diversified demands. In the last several decades, the development of optimal operating methodologies for reservoir operation has been very active. Yeh (1985) reviewed a number of approaches to reservoir operation and described the state of the art at that time. Since then, dynamic programming (DP) and its variants have been explored and frequently recommended to determine the optimal operating strategy for a multipurpose and/or multireservoir system (Kelman et al., 1990; Thomas et al., 1997; Perera and Codner, 1998; Chang et al., 2002; Chandramouli et al., 2002). More recently, fuzzy logic has been highly recommended for modelling reservoir operation to solve the inherent imprecision and vagueness characteristics in reservoirs. Even though a great number of promising results and considerable progress have been made, the research relating to real-time reservoir operation has still been very slow in finding its way into practice (Yeh, 1985; Russell and Campbell, 1996).

Intelligent control is a state-of-the-art technology that resembles the human thinking process in decision making and strategy learning, and it has been well recognized for its outstanding ability in controlling complex

\footnotetext{
* Correspondence to: Fi-John Chang, Department of Bioenvironmental Systems Engineering, National Taiwan University, Taipei, Taiwan. E-mail: changfj@ntu.edu.tw 


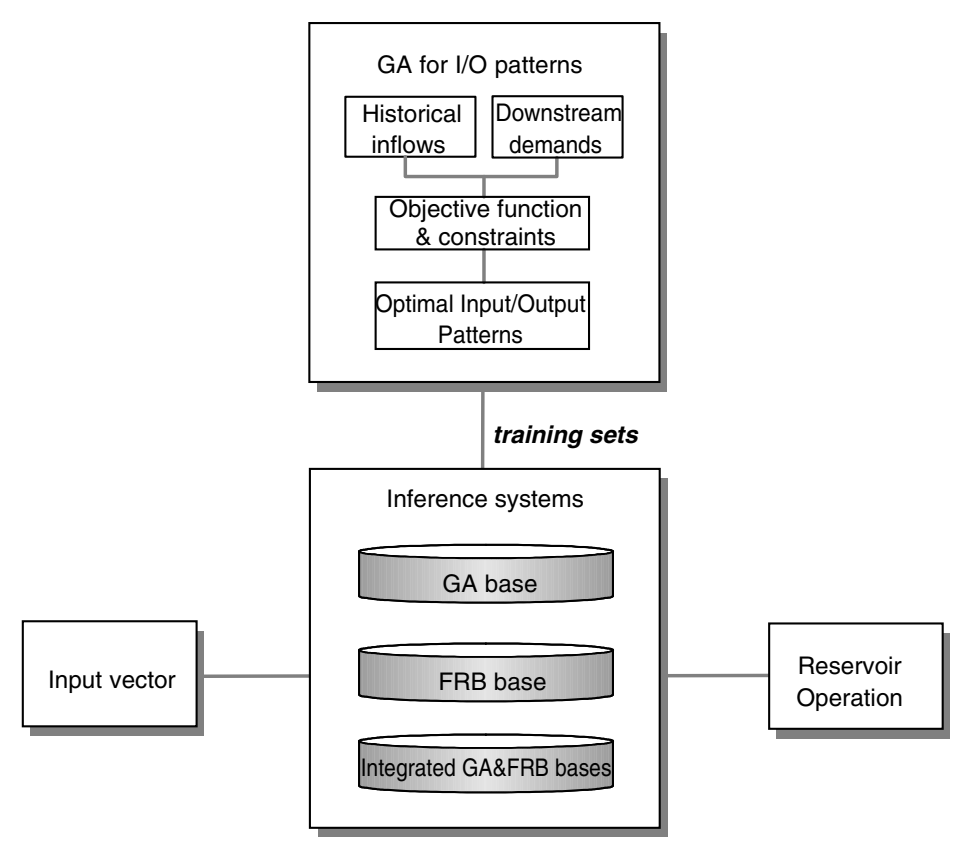

Figure 1. The framework of an intelligent control system for reservoir operation

systems, such as aerospace systems (Lin and Su, 2000), dynamic systems (Becerikli et al., 2003), and motor dynamic systems (Davies and Watton, 1995). In order to bridge the gap between academic research and actual operation, we proposed an intelligent control methodology for reservoir operation in our previous study. The intelligent control system combines two major procedures: a genetic algorithm (GA) and an adaptive network-based fuzzy inference system (ANFIS). The GA is used to search the optimal reservoir operating histogram based on a given inflow series, which can be recognized as the base optimal input-output training pattern for the intelligent control system. From these training patterns, the ANFIS is then built to create the fuzzy inference system, construct the suitable structure and parameters of the network, and then estimate the optimal water release according to the current reservoir watermark and inflow situation. In this study, the intelligent control system is enhanced through incorporating knowledge extracted from operating rule curves. The extracted knowledge is implemented as extra input information for the original ANFIS. It is our thought and hope, if the built intelligent control system integrated more knowledge, especially the currently used rules or information, it should be more intelligent and more likely applicable.

In the following sections, the methodology of an intelligent control system and its included theorems, i.e. GA and ANFIS, are first briefly introduced. A presentation of the methodology with different types of knowledge for constructing the inference system for reservoir operation then follows. The framework is shown in Figure 1. This shows that the GA is used to obtain the optimization input-output patterns, and then the inference systems, which include three different types of rule base (i.e. GA, fuzzy rule base (FRB), and an integrated GA with an FRB) are used to estimate the optimal reservoir operation. The results of different types of model are then presented and compared with those of the traditional M-5 rule operating curves. Finally, the study conclusions are given.

\section{INTELLIGENT CONTROL SYSTEM}

To build up an intelligent control system, one needs to acquire knowledge or information about the system first. The knowledge could be rules or guidelines that explicitly show the way a system responds to an input. 
Unfortunately, in most cases, no strict rules exist to guide the way. One alternative way is to collect the input-output data, and then try to analyse and/or classify the input-output relation. If a solid relation can be found, then it can be used for future operation when we face a similar input situation. In this study, we attempt to build an intelligent control system for reservoir operation through the ANFIS. Because there is no optimal input-output pattern for training the ANFIS, we first use the GA to search the optimal reservoir operation based on a set of series of historical inflow data. ANFIS are introduced as follows.

\section{The GA}

The GA, which was invented by Holland (1975), is based on Darwinian natural selection with the mechanisms of population genetics. Now in widespread use for computational evolution, it has also been applied to solve a great number of water resource problems (Wang, 1991; Chang and Chen, 1998; Mantawy et al., 1999; Cai et al., 2001). Since the GA tends to mimic some of the processes observed in natural evolution, the variables are first coded as a string (biologically referred to as chromosome) or a solution, and then a set of candidate solutions, called a population, is created. The evolution starts from coded solutions and proceeds from generation to generation through genetic operators: reproduction, crossover and mutation. To evaluate the suitability of the derived solution, an objective function is required. In each generation, we calculate the values of the objective function for each chromosome. Those with a high value of the objective function have high probability of reproducing into the next generation; those with a low value of the objective function do not. As the aforementioned genetic operators proceed, the optimal goal would eventually be achieved.

As mentioned above, the input-output patterns are crucial knowledge for training the intelligent control system. Because historical and/or optimal input-output patterns do not exist, we apply the GA to search the optimal reservoir release based on historical inflow series.

\section{The ANFIS}

The ANFIS was proposed by Jang (1993) and has been applied successfully to many problems (e.g. Djukanovic et al., 1997; Altug et al., 1999). This neuro-fuzzy network is a five-layer feedforward network that uses neural network learning algorithms coupled with fuzzy reasoning to map an input space to an output space. The major advantage of this network is that it can tune the complicated conversion of human intelligence to fuzzy systems. We notice that even though artificial neural networks (ANNs) have been quite successfully used in a great number of hydrological problems, there are only a few published works on reservoir operation using the ANN approach (e.g. Raman and Chandramouli, 1996; Jain et al., 1999; Hasebe and Nagayama, 2002). The greatest difficulty is probably due to lack of solid knowledge and/or reliable input-output patterns of reservoir operation.

The optimal input-output patterns obtained from the GA are used to construct the ANFIS framework. The details of the ANFIS algorithm can be found in Jang (1993) or Chang and Chang (2001). The simplified ANFIS architecture is shown in Figure 2, and a brief introduction of the model follows.

Layer 1: input nodes. Every node $i$ in this layer is used to perform a membership function

$$
\begin{array}{lll}
O_{1, i}=\mu_{A_{i}}(x) & \text { for } & i=1,2 \\
O_{1, i}=\mu_{B_{i-2}}(y) & \text { for } & i=3,4
\end{array}
$$

where $x$ and $y$ are the crisp inputs to node $i$, and $A_{i}$ and $B_{i}$ are the linguistic labels characterized by appropriate membership functions $\mu_{A_{i}}$ and $\mu_{B_{i}}$ respectively. The outputs $O_{1, i}$ are membership functions of $A_{i}$ and $B_{i}$.

Layer 2: rule nodes. T-norm operators are employed to perform the multiplications of the incoming signals to generate the outputs of this layer. The algebraic product is used and shown as follows:

$$
\begin{aligned}
O_{2, k} & =w_{k}=\mu_{A_{i}}(x) \times \mu_{B_{j}}(y) \\
k & =1, \ldots, 4 ; i=1,2 ; j=1,2
\end{aligned}
$$




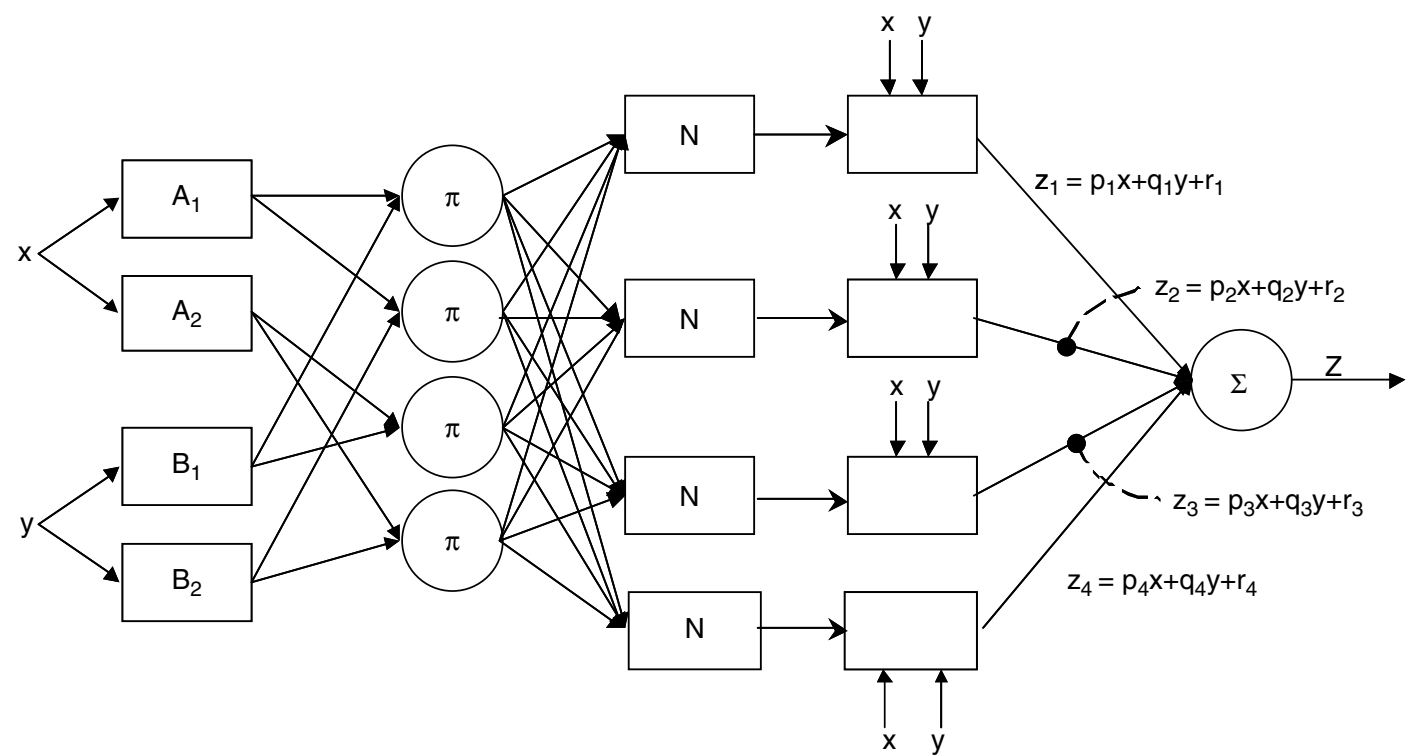

Figure 2. ANFIS architecture for a two-input Sugeno fuzzy model with four rules

Therefore, the outputs $O_{2, k}$ of this layer are the products of the corresponding degrees from layer 1 .

Layer 3: average nodes. The $i$ th node's output in this layer is the ratio of the $i$ th node's output from the previous layer to their total:

$$
O_{3, i}=\bar{w}_{i}=\frac{w_{i}}{\sum_{k=1}^{4} w_{k}} \quad i=1, \ldots, 4
$$

Layer 4: consequent nodes. In this layer, the $i$ th node corresponds to the following function:

$$
O_{4, i}=\bar{w}_{i} f_{i}=\bar{w}_{i}\left(p_{i} x+q_{i} y+r_{i}\right) \quad i=1, \ldots, 4
$$

where $\left\{p_{i}, q_{i}, r_{i}\right\}$ is the parameter set in the consequent part of the first-order Sugeno fuzzy model (Takagi and Sugeno, 1983).

Layer 5: output nodes. In this layer, a defuzzification inference is used to transform the fuzzy results of this model into a crisp output. Hence, the sum of all incoming signals is used to generate the decision crisp output:

$$
O_{5,1}=\sum_{i=1}^{4} \bar{w}_{i} f_{i}=\frac{\sum_{i=1}^{4} w_{i} f_{i}}{\sum_{i=1}^{4} w_{i}}
$$

\section{PRESENTATION OF CASE STUDY}

\section{Case study: Shihmen reservoir}

The Shihmen reservoir, located upstream of the Tahan River (Figure 3), is used as the example for the study. The reservoir operates for the purposes of water supply for irrigation, industrial and domestic uses, 


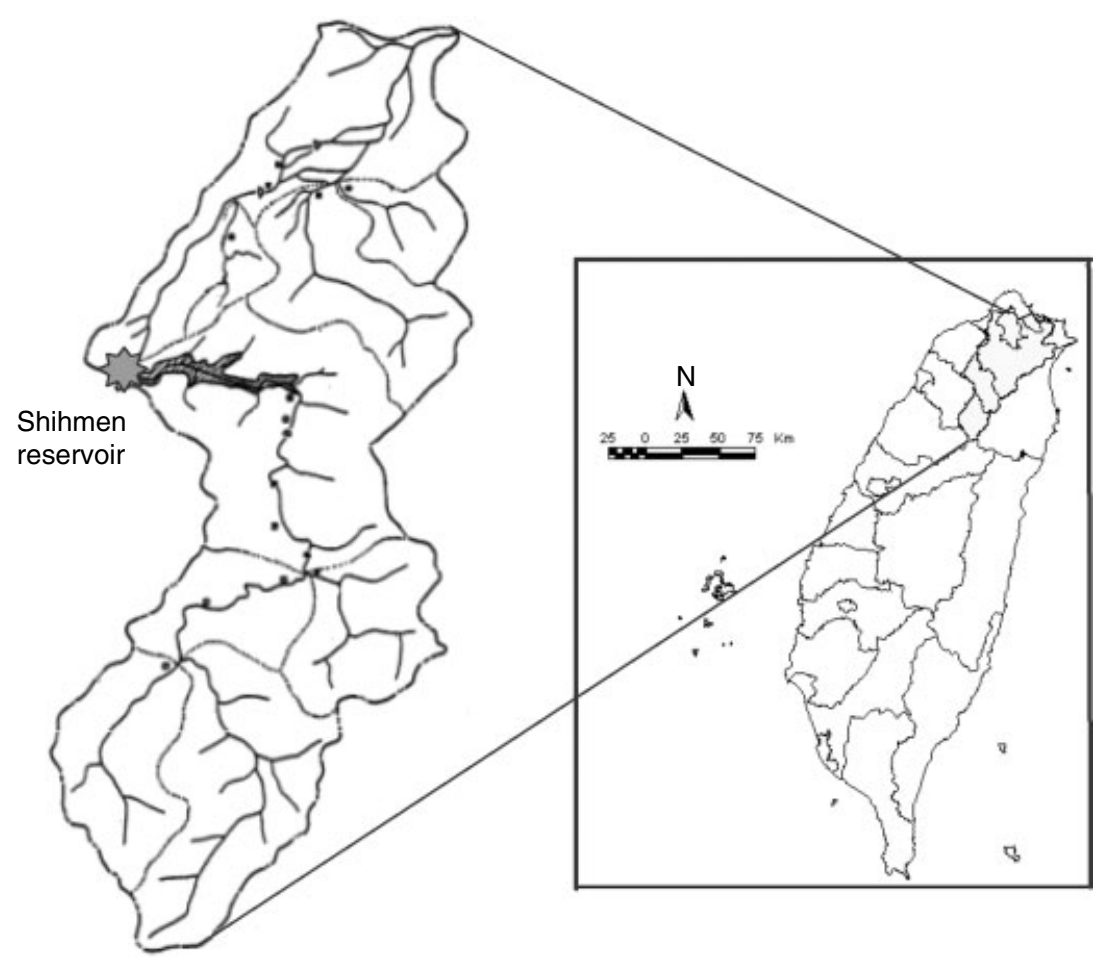

Figure 3. The location of Shihmen reservoir

flood control, hydropower generation, and recreation facilities. The watershed area is $763.4 \mathrm{~km}^{2}$ and its active storage volume is approximately $2.35 \times 10^{8} \mathrm{~m}^{3}$. Inflow data for the past 36 years are obtained. A period of 10 days, which is a traditional reference time frame in the Chinese agricultural society, is used as a time step. According to this scale, each month has three time steps, and every year has 36 steps. Consequently, we have $1296(36 \times 36)$ inflow records. In the case study, 1.2 times the water demand in the year of 2001 is adopted to represent the requirement for the simulation period, a total of $1329 \times 10^{6} \mathrm{~m}^{3} \mathrm{year}^{-1}$.

\section{The objective function and constraints}

Since water release from the reservoir is used to assess the long-term performance of reservoir operation in this study, we define the objective function of the reservoir operation as follows:

$$
\min (\text { ObjFunc })=\min \left\{\sum_{i=1}^{36}\left[\max \left(0, \frac{D_{i}-O_{i}}{D_{i}}\right)\right]^{2} \times n_{i}\right\}
$$

where $D_{i}$ is the water demand of the $i$ th 10 day period, $O_{i}$ is the outflow of the $i$ th 10 day period, and $n_{i}$ is the number of 10 day periods in a continuous deficit in the $i$ th 10 day period. The purpose of the variable $n_{i}$ in the objective function is to enlarge the effect of continuous deficiency and to avoid the occurrence of a continuous deficiency.

Moreover, the main task of the operator in reservoir operation is to meet the objectives as well as possible while complying with legal and other constraints. The mass balance equation and the physical or boundary conditions for reservoir operation are as follows: 
1. The mass balance equation (water balance at reservoir) or continuity equation is

$$
S_{i}=S_{i-1}+I_{i}-O_{i}
$$

where $S_{i}$ is the effective storage of the $i$ th 10 day period and $I_{i}$ is the inflow of the $i$ th 10 day period.

2. The initial storage $S_{0}=50$.

3. The variable $S_{i}$ can vary only between the maximum and the dead storage of the reservoir

$$
0 \leq S_{i} \leq 235 \cdot 745
$$

4. The conditions on the final stage of the reservoir storage are

$$
0 \cdot 9 S_{0} \leq S_{36} \leq 1 \cdot 1 S_{0}
$$

\section{The prior handling of data}

In this study, the 36 years of historical inflow data are divided into three independent sets: training, verification, and testing sets (Figure 4). To derive suitable input-output patterns, the GA and the rule-curves methods are applied.

GA for optimal input-output patterns. The historical inflow data are obtained from the Water Resource Agency, Taiwan. The GA is first employed to search the optimal release series based on a specific water demand series and the inflow series in each year during the training period (1966-88). There are several important features in the process of the GA:

1. Real-valued chromosomes are used to group the unknown variables together. The 36 10-day period reservoir releases in each year are the unknown variables.

2. A combination of objective function (Equation (1)) and constraints is set as the fitness function.

3. The GA parameters crossover probability, mutation rate, and population size are $0 \cdot 8,0 \cdot 1$, and 1000 respectively.

4. The terminal conditions are set when the fitness function has reached a value less than $0 \cdot 01$, or the process has been executed more than 6000 generations.

Owing to the large domain of searching space and the great number of unknown variables, the GA is difficult to converge to a satisfactory solution. In fact, in most cases it terminates at a minimum. To improve this situation, we designed initial multiple sets of feasible values for these variables. Accordingly, the 1000 initial sets of searching variables were divided into three different searching spaces as follows:

$1.10 \%$ of the initial sets are scattered randomly in the neighbourhood of water demand.

2. $10 \%$ of the initial sets are scattered randomly in the neighbourhood of inflow.

3. The remainder are scattered randomly in the original feasible solution domain.

As a result of this modification, the solutions have dramatically improved and approximately convergent solutions are achieved for most of the training years. For example, Figure 5 presents the variation of fitness values from generation to generation for the year 2000 to prove its convergence.

The optimal results of the GA and another result of operating by traditional M-5 rule curves (Figure 6) are summarized in Figure 7. It is found that the GA has a much better performance in terms of lower (generalized shortage index GSI; defined in Equation (2)) value than M-5. Consequently, the optimal reservoir operating histogram obtained from the GA can be recognized as the training pattern in the next step. 
(a)

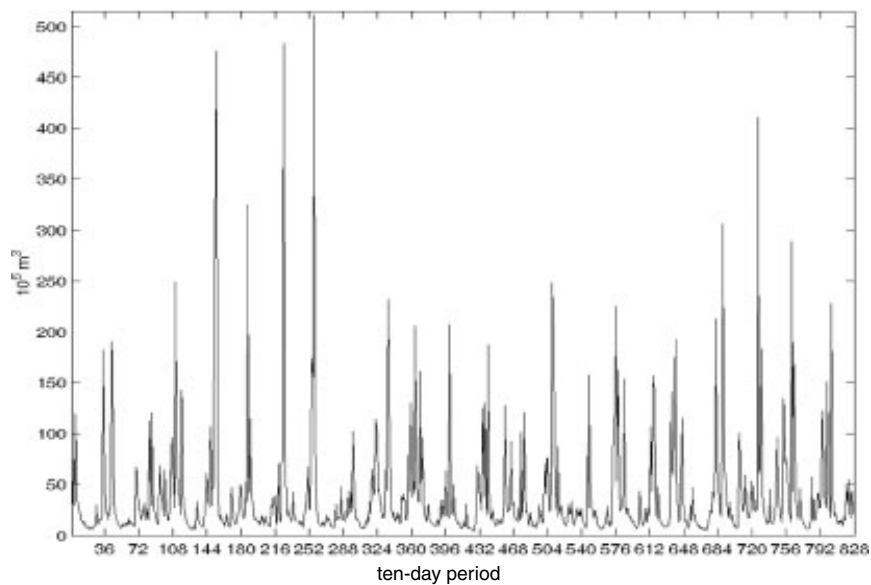

(b)

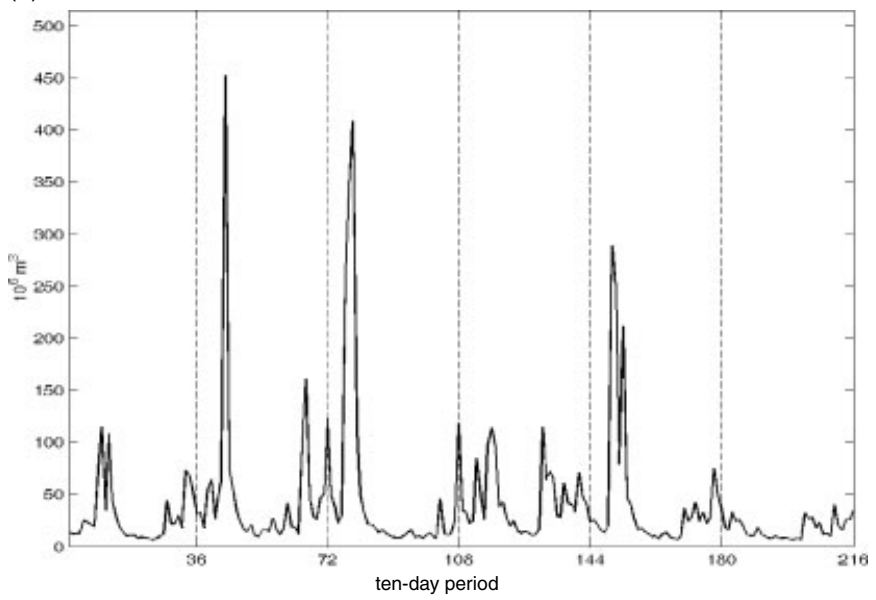

(c)

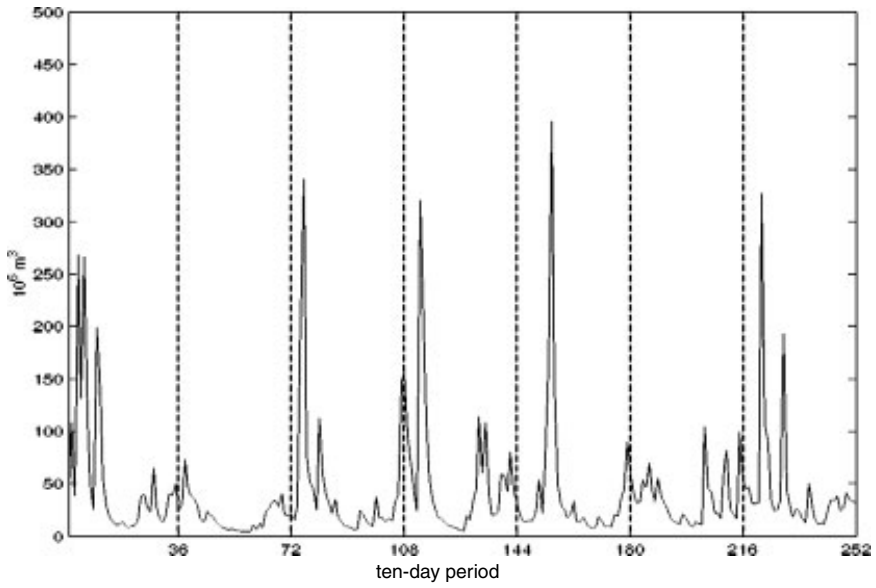

Figure 4. Three different procedural inflow time series: (a) the training procedure inflow time series; (b) the verification procedure inflow time series; (c) the testing procedure inflow time series 


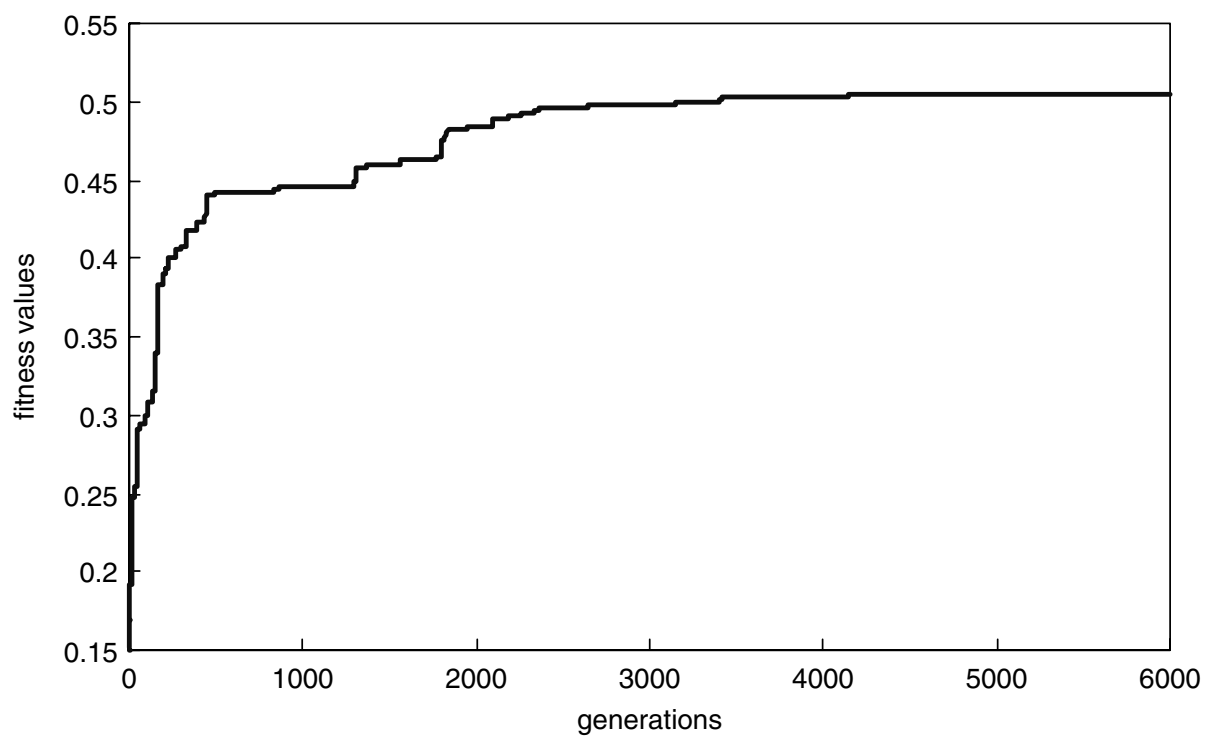

Figure 5. The variation of fitness values from generation to generation (year 2000)

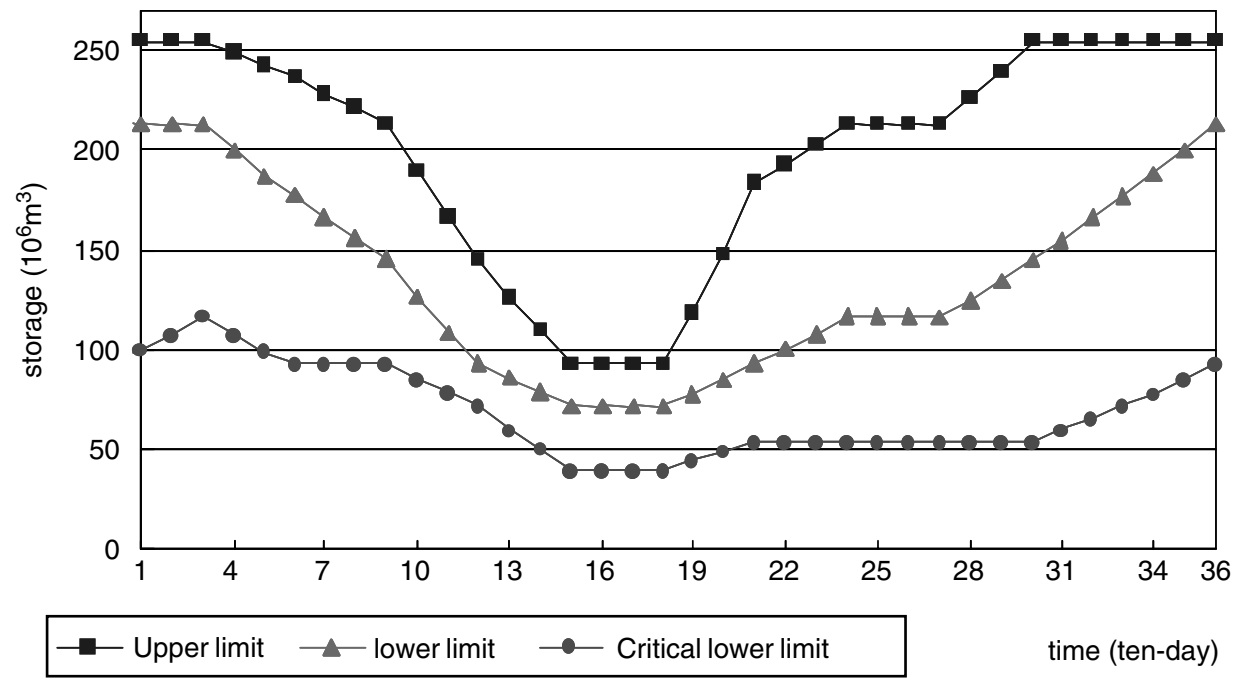

Figure 6. The M-5 rule curve of Shihmen reservoir

Fuzzified rule curve for reservoir operation. In Taiwan, most reservoirs are operated according to rule curves, which were obtained through intensity simulation analysis based on inflow data and water demands. Because the rule curve is the official way for guiding the reservoir operation, it is crucial to integrate this knowledge into the intelligent control system. According to the rule curves regulation, reservoir operation is mainly based on the water level and downstream water demand. For example, if time is in the $A_{1}$ period and water level $L$ is below the critical lower limit $B_{1}$, then water release $R$ is equal to $70 \%$ of water demand $D$. A set of rules can be constructed as follows:

Rule 1: $\operatorname{IF}\left(T=A_{1}\right.$ and $\left.L \leq B_{1}\right)$, THEN $\left(R=0 \cdot 7 D_{1}\right)$ 


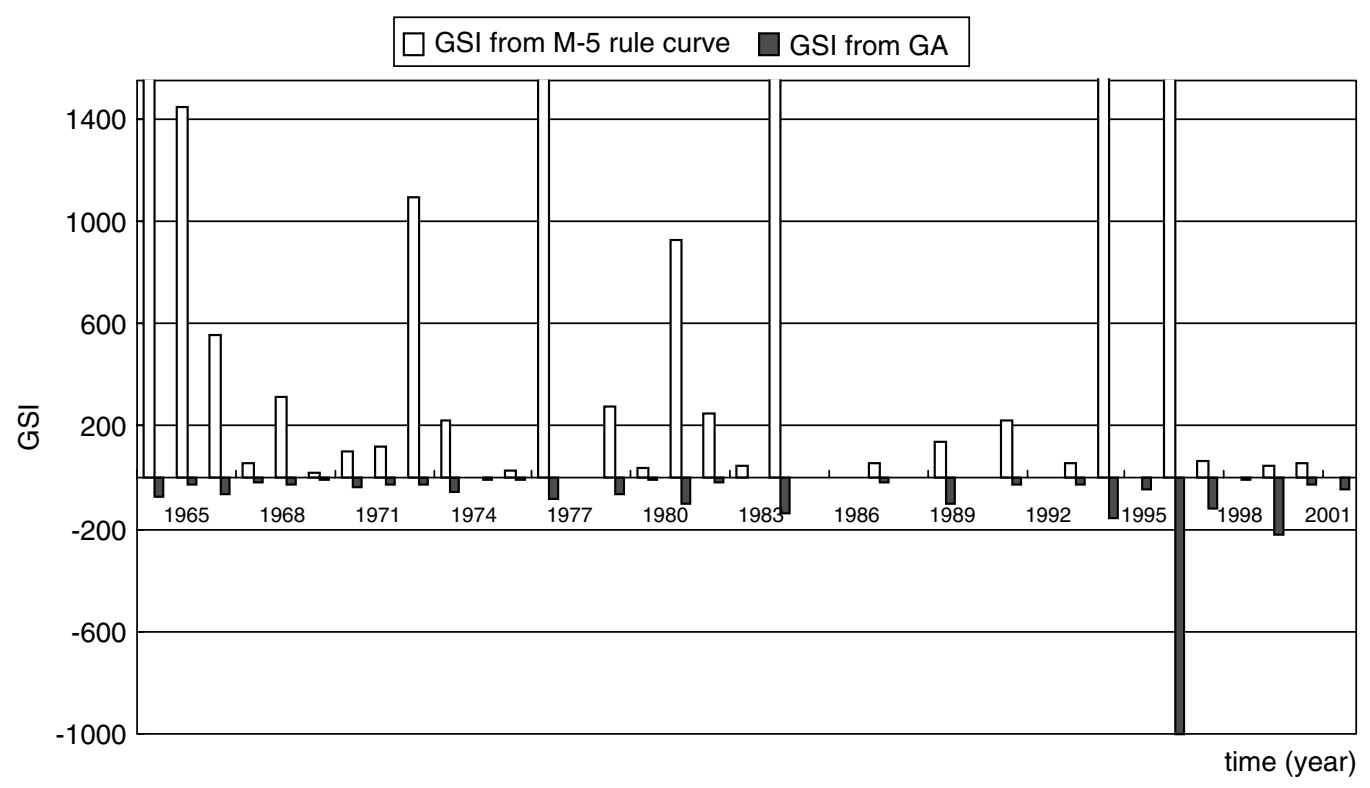

Figure 7. Comparison of GSI from the M-5 rule and the GA

Rule 2: $\operatorname{IF}\left(T=A_{2}\right.$ and $\left.L \leq B_{2}\right), \operatorname{THEN}\left(R=0 \cdot 7 D_{2}\right)$

Rule $k: \operatorname{IF}\left(T=A_{k}\right.$ and $\left.L \leq B_{k}\right), \operatorname{THEN}\left(R=0 \cdot 7 D_{k}\right)$

Because of the high variability of natural streamflow and the wide range between the curves, it is unlikely to determine the water release precisely according to the above rule alone. A knowledgeable expert with a lifetime of experience in reservoir operation is undoubtedly helpful and crucial for successfully operating the reservoir, especially in an extraordinary situation. However, expert experience and knowledge might not be obtained or be easily transformed.

Fuzzy logic, first introduced by Zadeh (1965), is gaining in popularity for modelling the ambiguity and uncertainty in the decision-making process and control theory. Recently, it has been applied successfully to various water resource problems (e.g. Russell and Campbell, 1996; Shrestha et al., 1996; Dou et al., 1999; Dubrovin et al., 2002). In this study, we attempted to incorporate the M-5 operating rule curves into our intelligent control system. The fuzzy logic provides a feasible way to transfer the rule curves into an FRB model. An FRB model is defined as the set of rules which consist of a set of input variables (such as the time of year and water level) or premises $A_{i, k}$ in the form of fuzzy sets with membership function $M_{i, k}$ and a set of consequence $R_{i}$ (i.e. water released to meet the various demands) also in the form of a fuzzy set. The mathematical form of the $i$ th rule is as follows:

$$
\text { If } a_{1} \text { is } A_{i, 1} \cdot a_{2} \text { is } A_{i, 2} \cdot \ldots \cdot a_{k} \text { is } A_{i, k} \text { then } R_{i}
$$

where $a_{k}$ is the $k$ th input variable and $A_{i, k}$ is the $k$ th variable premise condition, which has the form of a fuzzy set with membership function $M_{i, k}$, and $R_{i}$ is the $i$ th rule's consequence. Figure 8 shows the membership function of water level where, for instance, if time is in the $i$ th period and its water level is below the critical lower limit (60), water release $R_{i}$ is then equal to $70 \%$ of water demand $D_{i}$. 


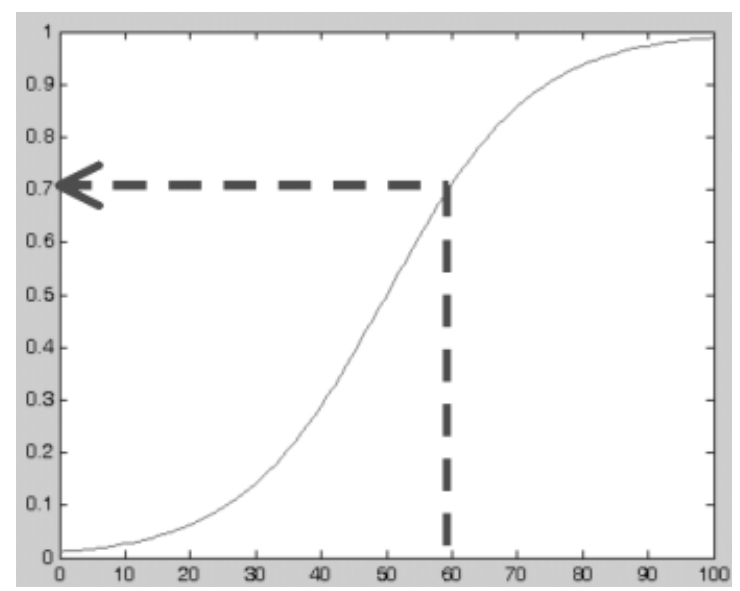

Figure 8. The membership function (sigmoid curve membership function) for water level

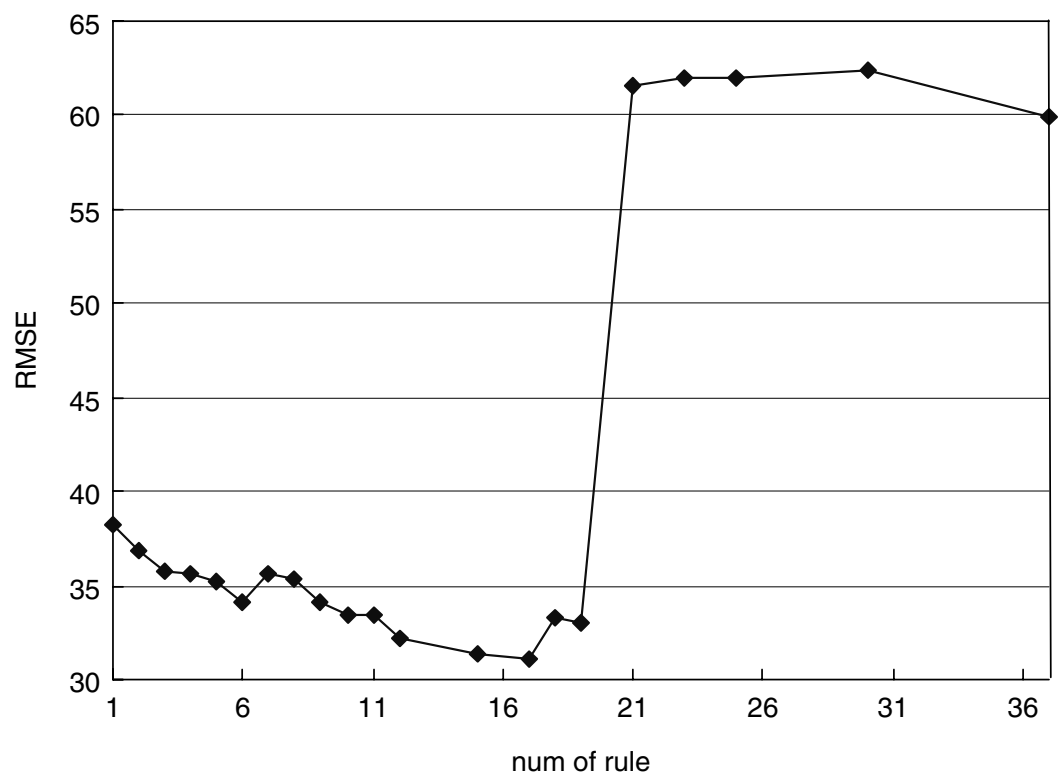

Figure 9. The relationship between root-mean-square error of the training procedure and the number of fuzzy rules in the GA base

\section{Application of the ANFIS}

Given the input-output patterns from (1) the GA and/or (2) the FRB, the ANFIS models are built to create the fuzzy inference system and then to estimate the optimal water release based on given input patterns.

1. In the GA case there are five input variables. Since each variable might have several values (in terms of the rule), and each rule includes several parameters of membership function in layer 2, there will be a huge number of parameters to determine as the rule is increased. To solve this problem, subtractive fuzzy clustering is used to establish the rule base relationship between the input and output variables (a detailed explanation can be found in Chang and Chang (2001)). Several clusterings have been evaluated. The results are shown in Figure 9, which indicates that 17 cluster rules can have lowest root-mean-square error. Since 
there are only 17 clusters, the inference system becomes quite simple. That is, as we give the five input variables' values, we can calculate their membership function value for each cluster, and then through the fuzzy inference system the result can be obtained.

2. In the FRB case we attempt to use the knowledge enclosed in the operating rule curves. In the M-5 rule curves, there is only one effective rule in each time step, and we have 36 time steps (10-day periods) in each year. There are 36 if-then rules as built in the previous section. To implement these rules within ANFIS we must have suitable output patterns for given input patterns. Because there is no actual corresponding output pattern for the input pattern, we use the optimal water release obtained from the GA as output patterns. Even though the input-output patterns are the same as the previous GA case, the rules, as is the knowledge, are different. The 36 fuzzy rules are implemented within ANFIS and their corresponding parameters and weights in each layer are systematically adjusted according to the training patterns.

\section{Integrating the GA and FRB rules}

In this case, we integrate the rules obtained from the GA (17 cluster rules) and the FRB (36 fuzzy rules) into a whole, so that the knowledge extracted from the input-output patterns by the GA and from operating rule curves by the FRB is all included. Again, there is no actual corresponding output pattern for a given input pattern. To train the ANFIS, we use the same output patterns obtained by the GA.

\section{RESULTS}

To construct and test the ANFIS model, the 36 years (1966-2001) of historical inflow data are divided into three independent sets: training, verification, and testing. The parameters and weights of these models are determined through an optimizing process based on a training data set. The most interesting system structure and corresponding parameters and weights are chosen based on the verification data set. Without any modification, the reliabilities of these systems are investigated using the testing data set.

For comparison, the simulation results of the M-5 curves and the optimal searching results by the GA are also given. The overall results for the M-5 curves, the GA, and different bases of the ANFIS models (namely the GA base, the FRB base, and the integrated GA with FRB base) are presented in Tables I and II. The results show that the ANFIS models could only be trained to obtain similar results to the GA method.

We believe that this is mainly because the optimal result obtained by the GA is based on being given the full series of input patterns to search the optimal operating strategy (output) for the series, whereas the ANFIS model is only based on previous and current $(t-1$ and $t)$ inflow and water-level information. Consequently, it is reasonable that the ANFIS model could only produce similar results to the GA method. The root-meansquare errors are within the range of 20 to 40 in all cases. To evaluate the model performance in terms of the

Table I. The results of the M-5, the GA and the three ANFIS models at different stages

\begin{tabular}{|c|c|c|c|c|c|c|c|c|c|}
\hline \multirow[t]{3}{*}{ Set } & \multirow[t]{3}{*}{ Year } & \multirow[t]{3}{*}{ M-5 GSI } & \multirow[t]{3}{*}{ GA GSI } & \multicolumn{6}{|c|}{ ANFIS $^{\mathrm{a}}$} \\
\hline & & & & \multicolumn{2}{|c|}{ GA base } & \multicolumn{2}{|c|}{ FRB base } & \multicolumn{2}{|c|}{ Integrated GA + FRB base } \\
\hline & & & & RMSE & GSI & RMSE & GSI & RMSE & GSI \\
\hline Training & $1966-88$ & $393 \cdot 96$ & $32 \cdot 83$ & 31.78 & $157 \cdot 52$ & $40 \cdot 17$ & 78.72 & $38 \cdot 90$ & $135 \cdot 00$ \\
\hline verification & 1989-94 & 981.95 & $54 \cdot 27$ & $27 \cdot 22$ & 237.76 & $34 \cdot 62$ & 297.71 & $36 \cdot 50$ & $185 \cdot 52$ \\
\hline Testing & $1995-2001$ & $633 \cdot 06$ & $66 \cdot 99$ & $30 \cdot 58$ & $171 \cdot 44$ & 32.97 & $130 \cdot 70$ & $29 \cdot 00$ & $47 \cdot 02$ \\
\hline Average & & $669 \cdot 66$ & $51 \cdot 36$ & $29 \cdot 86$ & 188.91 & 35.92 & 169.04 & $34 \cdot 80$ & $122 \cdot 51$ \\
\hline
\end{tabular}

${ }^{a}$ RMSE: root-mean-square error. 
Table II. The results of three ANFIS models and the M-5 rule curves in testing stage ${ }^{\mathrm{a}}$

\begin{tabular}{|c|c|c|c|c|c|c|c|c|c|c|c|c|}
\hline \multirow[t]{3}{*}{ Year } & \multicolumn{9}{|c|}{ ANFIS } & \multicolumn{3}{|c|}{ M-5 rule curves } \\
\hline & \multicolumn{3}{|c|}{ GA base } & \multicolumn{3}{|c|}{ FRB base } & \multicolumn{3}{|c|}{ Integrated GA + FRB base } & \multirow[t]{2}{*}{ (1) } & \multirow[t]{2}{*}{ (2) } & \multirow[t]{2}{*}{ GSI } \\
\hline & (1) & (2) & GSI & (1) & (2) & GSI & (1) & (2) & GSI & & & \\
\hline 1995 & 248.81 & 21 & $32 \cdot 30$ & $310 \cdot 01$ & 19 & $40 \cdot 06$ & 263.49 & 21 & $27 \cdot 26$ & 42.44 & 3 & 0.74 \\
\hline 1996 & $611 \cdot 22$ & 31 & $417 \cdot 26$ & $648 \cdot 44$ & 32 & 733 & $460 \cdot 80$ & 26 & $143 \cdot 16$ & 601.60 & 32 & $4256 \cdot 2$ \\
\hline 1997 & 358.99 & 21 & 249.08 & $248 \cdot 00$ & 18 & $16 \cdot 09$ & 272.65 & 22 & 37.46 & $218 \cdot 49$ & 12 & 65.95 \\
\hline 1998 & 258.42 & 17 & $32 \cdot 10$ & $187 \cdot 21$ & 16 & $5 \cdot 50$ & 229.62 & 19 & $22 \cdot 19$ & $35 \cdot 29$ & 8 & $3 \cdot 13$ \\
\hline 1999 & $457 \cdot 36$ & 21 & $378 \cdot 71$ & $372 \cdot 00$ & 21 & 43.76 & 415.49 & 24 & 82.74 & $290 \cdot 07$ & 14 & $49 \cdot 02$ \\
\hline 2000 & 329.76 & 24 & 44.69 & 294.21 & 23 & 38.27 & $244 \cdot 16$ & 18 & 9.75 & $119 \cdot 30$ & 13 & $56 \cdot 32$ \\
\hline 2001 & 291.90 & 23 & $45 \cdot 92$ & $258 \cdot 50$ & 19 & 38.59 & $164 \cdot 51$ & 16 & $6 \cdot 59$ & 11.46 & 1 & 0.00 \\
\hline Sum & $2556 \cdot 5$ & & & $2318 \cdot 4$ & & & $2050 \cdot 7$ & & & $1318 \cdot 6$ & & \\
\hline Average & & 23 & 171.4 & & $21 \cdot 14$ & $130 \cdot 70$ & & $20 \cdot 86$ & 47.02 & & 12 & $633 \cdot 1$ \\
\hline
\end{tabular}

a (1) Total water shortage $\left(10^{6} \mathrm{~m}^{3}\right)$. (2) The number of water shortages within the 36 periods of 1 year.

severity and length of water deficit, the GSI (Hsu, 1995) is used:

$$
\mathrm{GSI}=\frac{100}{N} \sum_{i=1}^{N}\left(\frac{\mathrm{DPD}_{i}}{100 \times \mathrm{DY}_{i}}\right)^{k}
$$

where DPD, the deficit percent 10-day period index, is given by

$$
\mathrm{DPD}=\sum(\mathrm{DDR}(\%) \times \mathrm{NDC})
$$

DDR is the 10-day period deficit rate, NDC is the number of 10-day periods in a continuous deficit, DY is the number of 10-day periods in a year (i.e. 36), $N$ is the number of sample years, and $k$ is a coefficient, usually taken as 2 .

From Table II, it is easy to see that the three ANFIS models produce a much better performance (in terms of small GSI values) than the M-5 curves in all cases. These results demonstrate that this new approach (with different knowledge), in comparison with the traditional M-5 rule curves, has superior performance with regard to the GSI.

From Tables I and II, we find that the results obtained from the GA-based and FRB-based ANFIS models are comparable, whereas the integrated GA and FRB ANFIS model does significantly reduce the GSI values in all cases. These results seem to provide interesting evidence that the ANFIS model has the ability to learn from the GA input-output pattern and from the FRB, and the system can be more intelligent if more information (i.e. integrated different kinds of knowledge) is implemented.

\section{CONCLUSIONS}

Intelligent control is a state-of-the-art technology that resembles the human thinking process, and its outstanding ability has been well recognized in controlling complex systems. Real-time reservoir operation is a continuous decision-making process under the inherent variability of natural phenomena. The operators must be based on an operating policy or predefined rules and cannot violate a large number of constraints, then must try to make things satisfactory for all the users. Apparently, this task can be very complex and difficult. 
The main purpose of this study is to enhance our previous intelligent control system through incorporating the knowledge extracted from the M-5 operating rule curves. The newly built intelligent control system includes two kinds of knowledge, i.e. the optimal input-output patterns obtained from the GA, and the FRB extracted from the rule curves.

The systems are implemented for the Shihmen reservoir, Taiwan, and its performance based on 36 years of historical streamflow data. The data are divided into three independent sets, namely training, verification, and testing. Three intelligent control systems are constructed through (1) an optimal input-output pattern obtained by the GA, (2) the FRB, and (3) the integrated GA and FRB frameworks. For the purpose of comparison, the simulation results by the traditional M-5 rule curve method were obtained.

The results demonstrate that all the intelligent control systems developed produce a much better performance (in terms of lower GSI values) than the M-5 rule curves. The intelligent control system can learn to make decisions for reservoir operation through (1) the optimal input-output training patterns obtained from the GA, (2) the FRB extracted from the M-5 rule curves regulation, and (3) the integration of these two different types of knowledge. Moreover, we find the models built (1) by the GA patterns and (2) by the FRB knowledge produce comparable performance, whereas the ANFIS model can be significantly improved if both types of knowledge are used to construct the intelligent control system. The results suggest that the ANFIS model has good ability to learn from different sources of knowledge, and the system can be more intelligent for reservoir operation, if more information is involved.

\section{ACKNOWLEDGEMENTS}

This paper is based on partial work supported by the National Science Council, R.O.C. (grant no. NSC 902313-B-002-323). In addition, we are indebted to the reviewers for their valuable comments and suggestions.

\section{REFERENCES}

Altug S, Chow MY, Trussell HJ. 1999. Fuzzy inference systems implemented on neural architectures for motor fault detection and diagnosis. IEEE Transactions on Industrial Electronics 46(6): 1069-1079.

Becerikli Y, Konar AF, Samad T. 2003. Intelligent optimal control with dynamic neural networks. Neural Networks 16(2): 251-259.

Cai XM, Mckinney DC, Lasdon LS. 2001. Solving nonlinear water management models using a combined genetic algorithm and linear programming approach. Advances in Water Resources 24(6): 667-676.

Chandramouli V, Kuppusamy KA, Manikandan K. 2002. Study on water sharing in a multi-reservoir system using a dynamic programmingneural network model. International Journal of Water Resources Development 18(3): 425-438.

Chang FJ, Chen L. 1998. Real-coded genetic algorithm for rule-based flood control reservoir management. Water Resource Management, EWRA 12(3): 185-198.

Chang FJ, Hui SC, Chen YC. 2002. Reservoir operation using grey fuzzy stochastic dynamic programming. Hydrological Processes 16: 2395-2408.

Chang LC, Chang FJ. 2001. Intelligent control of modeling of real time reservoir operation. Hydrological Processes 15: $1621-1634$.

Davies RM, Watton J. 1995. Intelligent control of an electrohydraulic motor drive system. Mechatronics 5(5): 527-540.

Djukanovic MB, Calovic MS, Vesovic BV, Sobajic DJ. 1997. Neuro-fuzzy controller of low head hydropower plants using adaptive-network based fuzzy inference. IEEE Transactions on Energy Conversion 12(4): 375-381.

Dou C, Woldt W, Bogardi I. 1999. Fuzzy rule-based approach to describe solute transport in the unsaturated zone. Journal of Hydrology $220(1-2): 74-85$.

Dubrovin T, Jolma A, Turunen E. 2002. Fuzzy model for real-time reservoir operation. Journal of Water Resources Planning and Management 128(1): $66-73$.

Hasebe M, Nagayama Y. 2002. Reservoir operation using the neural network and fuzzy systems for dam control and operation support. Advances in Engineering Software 33(5): 245-260.

Holland JH. 1975. Adaptation in Natural and Artificial Systems, 2nd edn. Massachusetts Institute of Technology: Cambridge, MA.

Hsu SK. 1995. Shortage indices for water-resources planning in Taiwan. Journal of Water Resources Planning and Management 121(2): $119-131$.

Hydrologic Engineering Center. 1996. Reservoir yield, generalized computer program 23-J2-L245. US Army, Corps of Engineers, Davis, CA.

Jain SK, Das A, Srivastava DK. 1999. Application of ANN for reservoir inflow prediction and operation. Journal of Water Resources Planning and Management 125(5): 263-271.

Jang J-SR. 1993. ANFIS: adaptive-network-based fuzzy inference system. IEEE Transactions on Systems, Man, and Cybernetics 23: 665-685. 
Kelman J, Stedinger JR, Copper LA, Hsu E, Yuan SQ. 1990. Sampling stochastic dynamic programming applied to reservoir operation. Water Resources Research 26(3): 447-454.

Lin CL, Su HW. 2000. Intelligent control theory in guidance and control system design: an overview. Proceedings of the National Science Council, ROC 24(1): 15-30.

Mantawy AH, Abdel-Magid YL, Selim SZ. 1999. A new genetic-based Tabu search algorithm for unit commitment problem. Electric Power Systems Research 49(2): 71-78.

Perera BJC, Codner G. 1998. Computational improvement for stochastic dynamic programming models of urban water supply reservoirs. Journal of the American Water Resources Association 34(2): 267-278.

Raman H, Chandramouli V. 1996. Deriving a general operating policy for reservoirs using neural network. Journal of Water Resources Planning and Management 122(5): 342-347.

Russell SO, Campbell PF. 1996. Reservoir operating rules with fuzzy programming. Journal of Water Resources Planning and Management 122(3): $165-170$.

Shrestha BP, Duckstein L, Stakhiv EZ. 1996. Fuzzy rule-based modeling of reservoir operation. Journal of Water Resources Planning and Management 122(4): 262-269.

Takagi T, Sugeno M. 1983. Fuzzy identification of systems and its applications to modeling and control. IEEE Transactions on Systems, Man, and Cybernetics 15: 116-132.

Thomas LC, Archibald TW, McKinnon KIM. 1997. An aggregate stochastic dynamic programming model of multiple reservoir systems. Water Resources Research 33: 333-340.

Wang QJ. 1991. The genetic algorithm and its application to calibrating conceptual rainfall-runoff models. Water Resources Research 27: 2467-2471.

Yeh W. 1985. Reservoir management and optimization models: a state of the art review. Water Resources Research 21: 1797-1818.

Zadeh LA. 1965. Fuzzy sets. Information and Control 8: 338-353. 